BI-TP 2001/05

\title{
On $e^{+} e^{-} \rightarrow W^{+} W^{-} \rightarrow 4 f(+\gamma)$ at LEP2
}

\author{
Yoshimasa Kurihara \\ KEK, Tsukuba, Japan \\ Masaaki Kuroda \\ Meijigakuin University, Yokohama, Japan \\ Dieter Schildknecht \\ Fakultät für Physik, Universität Bielefeld \\ Bielefeld, Germany
}

\begin{abstract}
The results on $e^{+} e^{-} \rightarrow W^{+} W^{-} \rightarrow 4 f(+\gamma)$ obtained by different groups are compared with each other. Differences in the results for the total cross section of up to about $0.6 \%$ are traced back to different ways of implementing the double-pole approximation.
\end{abstract}

*Suppported by the BMFT, Bonn, Germany, Contract 05 HT9PBA2 
In the workshop report on $W$-pair production at LEP2 [1], theoretical results on the cross section for the reaction $e^{+} e^{-} \rightarrow W^{+} W^{-} \rightarrow 4 f(+\gamma)$ from several working groups are presented. While the workshop has been in progress, based on our previous work [2], we obtained results for the total cross section of the above reaction that were approximately $0.5 \%$ larger than the results published in ref. [1]. In the present note, we give our results, which remained unpublished so far, and we compare them with the results of the $\mathrm{BBC}$ [3] and the RACOON [4, 5, 6] group in ref. [1].t]

We will arrive at the conclusion that different ways of implementing the double-pole approximation (DPA) that enters the evaluation of the cross section may lead to differences up to about $0.6 \%$ in the total cross section for the above-mentioned reaction.

We briefly summarize our approach [2] that will be referred to as KKS in the subsequent discussion of the different approaches. The total cross section is written as the sum of the Born cross section, $\sigma_{B}^{\text {full }}$, the virtual one-loop and soft-photon-radiation cross section, $\sigma_{V+S}$, and the hard-photon-radiation cross section, $\sigma_{H}$,

$$
\sigma_{\text {tot }}=\sigma_{B}^{\text {full }}+\sigma_{V+S}+\sigma_{H} .
$$

As in ref. [1], the Born cross section, $\sigma_{B}^{\text {full }}$, is based on the complete set of Feynman diagrams contributing to $e^{+} e^{-} \rightarrow$ four fermions, i.e. diagrams not containing $W$-resonance enhancements are also taken into account.

The expression for $\sigma_{V+S}$ is given by

$$
\begin{aligned}
\sigma_{V+S}= & \frac{1}{2 s}(2 \pi)^{-7} \frac{1}{512} \sum^{\prime} \int d k_{+}^{2} d k_{-}^{2} d \cos \theta_{W} d \hat{\varphi}_{2} d \cos \hat{\theta}_{2} d \hat{\varphi}_{4} d \cos \hat{\theta}_{4} \frac{\left|\overrightarrow{k_{ \pm}}\right|}{E_{\text {beam }}}(2) \\
& \left.\frac{\left|\mathcal{H}\left(e^{+}\left(\sigma_{+}\right) e^{-}\left(\sigma_{-}\right) \rightarrow W_{\lambda_{+}}^{+}\left(f_{1}\left(\tau_{1}\right) \bar{f}_{2}\left(\bar{\tau}_{2}\right)\right) W_{\lambda_{-}}^{-}\left(f_{4}\left(\tau_{4}\right) \bar{f}_{3}\left(\bar{\tau}_{3}\right)\right)\right)\right|^{2}}{\left[\left(k_{+}^{2}-M_{W}^{2}\right)^{2}+\Gamma_{W}^{2} M_{W}^{2}\right]\left[\left(k_{-}^{2}-M_{W}^{2}\right)^{2}+\Gamma_{W}^{2} M_{W}^{2}\right]}\right|_{\mathcal{O}(\alpha)},
\end{aligned}
$$

where $\sum^{\prime}$ indicates the spin sum over the final fermions as well as the spin average of the initial $e^{+} e^{-}$pair. The variables

$$
k_{+}^{2}, k_{-}^{2}, \cos \theta_{W}
$$

stand for the squared invariant masses and the cosine of the polar production angle of the fermion pairs in the $e^{+} e^{-}$center-of-mass frame, while

$$
\hat{\varphi}_{2}, \cos \hat{\theta}_{2} ; \hat{\varphi}_{4}, \cos \hat{\theta}_{4}
$$

denote the fermion angles in the rest frames of the pairs of invariant masses $k_{+}^{2}$ and $k_{-}^{2}$, respectively; specifically, in the case of on-shell production, we

\footnotetext{
${ }^{1}$ Additional results, not published in refs. [1, 3, 5n, were kindly provided to us by the $\mathrm{BBC}$ group (Beenakker, Berends and Chapovsky) and the RACOON group (Denner, Dittmaier, Roth and Wackeroth).
} 
have $k_{+}^{2}=k_{-}^{2}=M_{W}^{2}$. As indicated by the integration variables in (2), the amplitude describing (on-shell or off-shell) $W$ production and decay in (2) is to be expressed in terms of $k_{+}^{2}, k_{-}^{2}, \cos \theta_{W}$ and the fermion-pair rest-frame angles $\hat{\varphi}_{2}, \cos \hat{\theta}_{2}$ and $\hat{\varphi}_{4}, \cos \hat{\theta}_{4}$. The choice of the rest-frame angles in (2), in view of the DPA, is dictated by the requirement that the off-shell and on-shell decay configurations have identical (normalized) decay probabilities.'2 The DPA now consists of ignoring any additional dependence on $k_{ \pm}^{2}$ in the $W$ boson (production and decay) amplitude in (2) by evaluating the amplitude at the pole position, $k_{+}^{2}=k_{-}^{2}=M_{W}^{2}$. The variation of the phase space with $k_{ \pm}^{2}$ in $(2)$ is taken into account via the dependence of the $W$-boson three momentum, $\left|\vec{k}_{ \pm}\right|$, that is given by

$$
\left|\vec{k}_{ \pm}\right|^{2}=\frac{1}{4 s}\left(s^{2}+k_{+}^{4}+k_{-}^{4}-2 s\left(k_{+}^{2}+k_{-}^{2}\right)-2 k_{+}^{2} k_{-}^{2}\right),
$$

where the $e^{+} e^{-}$center-of-mass energy squared is denoted by $s$.

Also the hard-photon radiation is treated in the DPA, the expression for $\sigma_{H}$ being analogous to (2).

The RACOON ansatz, when evaluating $\sigma_{V}$ in the DPA according to (2), differs from ours in their choice of the laboratory-frame fermion angles. This implies that in general off-shell and on-shell amplitudes at different restframe angles are associated with each other, and accordingly an avoidable difference between the off-shell amplitude and its approximating on-shell one is introduced. The choice of the laboratory angles also implies a dependence on which pair of outgoing leptons (quarks) is used to specify the four-fermion configuration. Fortunately, as noted by RACOON, using different pairs of fermions affects the result for the total cross section only at the level of $0.1 \%$ [6].

In the treatment of the BBC group, also the phase space and the propagators in (2) are evaluated on the $W$ mass shell, $k_{ \pm}^{2}=M_{W}^{2}$, by carrying out the substitution

$$
\frac{M_{W} \Gamma_{W}}{\left(k_{ \pm}^{2}-M_{W}^{2}\right)+M_{W}^{2} \Gamma_{W}^{2}} \longrightarrow \pi \delta\left(k_{ \pm}^{2}-M_{W}^{2}\right)
$$

that becomes exact in the zero-width limit] of $M_{W} \Gamma_{W} \rightarrow 0$. As, by construction, there is no off-shell phase-space element in the BBC ansatz, the question of which on-shell amplitude is to be associated with a certain offshell phase-space element becomes meaningless.

Concerning the photon radiation, the RACOON treatment differs from the DPA ones of BBC and KKS by taking into account radiation from the full set of Born diagrams for $e^{+} e^{-} \rightarrow 4$ fermions.

\footnotetext{
${ }^{2}$ The (normalized) rest-frame decay distribution is independent of the virtuality, i.e. independent of whether $k_{ \pm}^{2}=M_{W}^{2}$ or $k_{ \pm}^{2} \neq M_{W}^{2}$.

${ }^{3}$ In ref.[2], such an approximation is called the zero-width approximation.
} 


\begin{tabular}{|l|c|c|c|}
\hline \multirow{2}{*}{ Group } & \multicolumn{2}{|c|}{ DPA } & photon radiation \\
\cline { 2 - 3 } & phase space and fermion angles & \\
\hline \multirow{2}{*}{ BBC } & \multicolumn{2}{|c|}{ zero-width approximation } & \multirow{2}{*}{ DPA } \\
\cline { 2 - 3 } & on-shell & irrelevant & \\
\hline KKS & off-shell & $W^{ \pm}$rest frames & DPA \\
\hline RACOON & off-shell & laboratory frame & full \\
\hline
\end{tabular}

Table 1. Comparison of the schemes of computing radiative corrections by three groups.

Finally, we note that RACOON in its treatment includes non-factorizable radiative corrections as well. Since such corrections cancel [7, 8, 9, 10] in the total cross section, they were not included in the KKS evaluation. In view of the discussion of differences between the final results of the different groups below, we note that RACOON did not explicitly verify the cancellation of the non-factorizable radiative corrections in the sum of $\sigma_{V}, \sigma_{S}$ and $\sigma_{H}$. The non-factorizable radiative corrections taken into account by $\mathrm{BBC}$, by construction, drop out in the total cross section.

We schematically summarize the assumptions underlying the approaches by the different groups in Table 1 .

In our calculation, as in ref.[1], the $\alpha_{G_{\mu}}$ scheme is used, i.e. the coupling constants appearing at tree level are evaluated in terms of the muon decay constant, $G_{\mu}$. In order to make the comparison of the results of the different groups transparent, we stick to comparing cross sections with order $\alpha$ corrections, discarding the inclusion of higher-order effects, such as the exponentiation of the initial state radiation, etc.

We use the set of input parameters also employed in the LEP2 workshop proceedings [1],

$$
\begin{aligned}
M_{Z} & =91.1867 \mathrm{GeV}, \quad M_{W}=80.35 \mathrm{GeV}, \quad M_{H}=150 \mathrm{GeV}, \\
G_{\mu} & =1.166389 \times 10^{-5} \mathrm{GeV}^{-2}, \quad \Gamma_{W}=2.08699 \mathrm{GeV} .
\end{aligned}
$$

The numerical integrations were performed by using the Monte Carlo program BASES [11].

Various checks of our program were performed:

i) The cancellation of the ultraviolet and the infrared divergences in the cross section was confirmed analytically as well as numerically.

ii) The stability against changing the soft-photon separation energy, $k_{c}$, was checked by varying $k_{c}$ between $0.001 \mathrm{GeV}$ and $0.1 \mathrm{GeV}$.

iii) The cancellation of mass singularities in the total cross section was checked by varying the fermion masses.

iv) The virtual correction, $\sigma_{V}$, and the soft photon radiation, $\sigma_{S}$, were independently calculated by using GRACE [12 as well as the program constructed by one of the present authors 13 several years ago. 


\section{The Leptonic $W^{ \pm}$decay}

We start with the leptonic decay, $e^{+} e^{-} \rightarrow W^{+} W^{-} \rightarrow\left(\bar{\mu}, \nu_{\mu}\right)\left(\tau, \bar{\nu}_{\tau}\right)(+\gamma)$. In Tables 2 to 4 , our results (KKS) are compared with the ones from BBC and RACOON. In order to explicitly verify that our numerical evaluation is consistent with the one from $\mathrm{BBC}$, we have also performed an evaluation á la $\mathrm{BBC}$, i.e. by replacing the full phase space in (2) by the zero-width approximation (5). Compare KKS (zero-width) in Tables 2 to 4.

\begin{tabular}{|c|l|l|l|}
\hline Group & $\sqrt{s}=184 \mathrm{GeV}$ & $\sqrt{s}=189 \mathrm{GeV}$ & $\sqrt{s}=200 \mathrm{GeV}$ \\
\hline BBC & & & 200.14 \\
KKS(zero-width) & $181.84(9)$ & $190.67(9)$ & $200.14(9)$ \\
KKS & $182.80(9)$ & $191.40(9)$ & $200.57(9)$ \\
RACOON & $182.53(11)$ & $190.96(11)$ & $199.98(12)$ \\
\hline
\end{tabular}

Table 2. The total cross section for the process $e^{+} e^{-} \rightarrow W^{+} W^{-} \rightarrow\left(\bar{\mu} \nu_{\mu}\right)\left(\tau \bar{\nu}_{\tau}\right)$ with strict order-alpha corrections evaluated by BBC, KKS and RACOON at $\sqrt{s}=184,189$ and 200 $\mathrm{GeV}$.

\begin{tabular}{|c|c|c|l||c|c|}
\hline Group & \multicolumn{1}{|c|}{$\sigma_{B}^{\text {full }}$} & $\sigma_{V+S+H}$ & \multicolumn{1}{c|}{$\sigma_{\text {tot }}$} & $\sigma_{V}^{\text {fact }}$ & $\sigma_{V+S+H^{-}} \sigma_{V}^{\text {fact }}$ \\
\hline BBC & $219.99(5)$ & -19.85 & 200.14 & & \\
KKS(zero-width) & $219.85(7)$ & -19.71 & $200.14(9)$ & -437.92 & 418.21 \\
KKS & $219.85(7)$ & -19.28 & $200.57(9)$ & -422.87 & 403.59 \\
RACOON & $220.06(6)$ & -20.08 & $199.98(12)$ & -422.75 & 402.67 \\
\hline
\end{tabular}

Table 3. The full-Born cross section, $\sigma_{B}^{\text {full }}$, as well as the radiative correction, $\sigma_{V+S+H}$, adding up to the total cross section, $\sigma_{t o t}$, for the process $e^{+} e^{-} \rightarrow W^{+} W^{-} \rightarrow\left(\bar{\mu} \nu_{\mu}\right)\left(\tau \bar{\nu}_{\tau}\right)$ at $\sqrt{s}=200 \mathrm{GeV}$. The factorizable part of the virtual correction, $\sigma_{V}^{\text {fact }}$, as well as the difference, $\sigma_{V+S+H^{-}} \sigma_{V}^{\text {fact }}$, for $m_{\gamma}=10^{-6} \mathrm{GeV}$ is also shown.

\begin{tabular}{|c|l|c|c|c|}
\hline Group & $\sigma_{B}^{\mathrm{DPA}}$ & $R_{B}^{\mathrm{DPA}}$ & $\sigma_{V+S+H}$ & $R_{V+S+H}^{\mathrm{DPA}}$ \\
\hline BBC & $229.8(1)$ & $3.5 \%$ & -19.85 & $3.0 \%$ \\
KKS(zero-width) & $229.58(2)$ & $3.4 \%$ & -19.71 & $2.2 \%$ \\
KKS & $221.72(4)$ & & -19.28 & \\
\hline
\end{tabular}

Table 4. The DPA-Born cross section, $\sigma_{B}^{\text {DPA }}$, as well as the order $\alpha$ correction due to virtual effects, soft and hard bremsstrahlung, $\sigma_{V+S+H}$, for the process $e^{+} e^{-} \rightarrow W^{+} W^{-} \rightarrow$ $\left(\bar{\mu} \nu_{\mu}\right)\left(\tau \bar{\nu}_{\tau}\right)(+\gamma)$ at $\sqrt{s}=200 \mathrm{GeV}$, and their relative changes as a result of replacing the off-shell phase space by the zero-width approximation. The relative changes $R_{B}^{\mathrm{DPA}}$ and $R_{V+S+H}^{\mathrm{DPA}}$ are defined by $R_{B}^{\mathrm{DPA}}=\frac{\sigma_{B}^{\mathrm{DPA}}(B B C)-\sigma_{B}^{\mathrm{DPA}}(K K S)}{\sigma_{B}^{\mathrm{DPA}}(B B C)}$ etc.

In the interpretation of the results in Tables 2 to 4 , we proceed in several steps:

i) Comparing the results of KKS (zero-width) and BBC, in Tables 2 and 3, we find good agreement between these independent calculations of $\sigma_{\text {tot }}$ and the virtual correction $\sigma_{V+S+H}$. The comparison explicitly demonstrates that both calculations, $\mathrm{BBC}$ and $\mathrm{KKS}$, are reliable, elementary

\footnotetext{
${ }^{4}$ We note that all cross sections displayed in Tables 2 to 8 are given in units of $f b$.
} 
errors being excluded. The relative difference between the BBC (the $\mathrm{KKS}$ (zero-width)) and the KKS results is displayed in Table 4. Since the virtual corrections are proportional to the Born cross section evaluated in DPA, the relative change in $\sigma_{V+S+H}$ of about $3 \%$ is approximately given by the relative change in $\sigma_{B}^{\mathrm{DPA}}$. The relative change of around $3 \%$ amounts to a decrease of the total cross section by about $0.3 \%$ or $0.6 \mathrm{fb}$ (compare Table 2 ) of KKS(zero-width) relative to KKS.

We conclude that the $0.3 \%$ reduction of $\sigma_{\text {tot }}$ of $\mathrm{BBC}$ relative to KKS is due to the use of the simplifying zero-width approximation that enlarges $\sigma_{B}^{\text {DPA }}$ and accordingly the absolute value of the (negative) radiative correction, $\sigma_{V+S+H}$.

ii) We turn to a discussion of the differences between the results of KKS and RACOON. According to Table 1 , the increase of $0.6 \mathrm{fb} \cong 0.3 \%$ in Tables 2 and 3 of $\sigma_{\text {tot }}$ from KKS relative to $\sigma_{\text {tot }}$ from RACOON at $\sqrt{s}=200 \mathrm{GeV}$ is to be traced back to differences in the (soft and hard) photon radiation? KKS treats photon radiation in the DPA, while RACOON radiates photons from all diagrams. Table 3 indeed shows that the virtual factorizable corrections, $\sigma_{V}^{\text {fact }}$, of KKS and RACOON agree very well, the differences in $\sigma_{V+S+H}$ and $\sigma_{\text {tot }}$ thus indeed being due to the different treatment of the radiation. Compare the value of $\sigma_{V+S+H}-\sigma_{V}^{\text {fact }}$ in Table 3 .

iii) Finally, we compare the results of BBC and RACOON. As noted in Table 1 , the $\mathrm{BBC}$ procedure differs from the one by RACOON in applying the DPA to the radiation in conjunction with the use of the zero-width approximation. Since the zero-width approximation decreases the total cross section, the increase of the cross section of $\mathrm{BBC}$ relative to RACOON induced by applying the DPA to the treatment of the radiation is (partially) cancelled. The surprisingly good agreement between $\sigma_{\text {tot }}$ by RACOON and by BBC at $200 \mathrm{GeV}$ appears as an accidental one. In this connection it is to be noted that the difference between RACOON and BBC, or rather KKS (zero-width), according to Table 2 , changes with decreasing $e^{+} e^{-}$energy from $+0.16 f b$ to $-0.7 f b$.

\section{The Hadronic $W^{ \pm}$decay}

For the hadronic channel, $e^{+} e^{-} \rightarrow W^{+} W^{-} \rightarrow(u \bar{d})(\bar{c} s)$, no results from $\mathrm{BBC}$ are available. We compare our results, KKS and KKS(zero-width), with the result from RACOON.

\footnotetext{
${ }^{5}$ The discussion is simplified, in so far as we ignore the effect of the different choices of the fermion angles and assume exact cancellation of the non-factorizable corrections in the RACCON treatment. Compare the more detailed discussion in the case of hadronic $W^{ \pm}$decays below.
} 
In our calculations, we used values of the quark masses that coincide with the ones from RACOON (in units of $\mathrm{GeV} / \mathrm{c}^{2}$ ),

$$
\begin{array}{lll}
m_{u}=0.0485, & m_{c}=1.55, & m_{t}=174.17 \\
m_{d}=0.0485, & m_{s}=0.15, & m_{b}=4.5 .
\end{array}
$$

The quark masses enter $\sigma_{V}, \sigma_{S}$ and $\sigma_{H}$ in terms of mass-singular logarithms, but are obviously irrelevant for the total cross section.

\begin{tabular}{|c|c|c|c|}
\hline Group & $\sqrt{s}=184 \mathrm{GeV}$ & $\sqrt{s}=189 \mathrm{GeV}$ & $\sqrt{s}=200 \mathrm{GeV}$ \\
\hline KKS(zero-width) & $1636.9(9)$ & $1716.0(9)$ & $1801.5(9)$ \\
KKS & $1647.5(9)$ & $1724.7(9)$ & $1807.6(9)$ \\
RACOON & $1640.7(7)$ & $1716.4(8)$ & $1797.4(9)$ \\
\hline
\end{tabular}

Table 5. The comparison of the total cross section for the process $e^{+} e^{-} \rightarrow(u \bar{d})(\bar{c} s)(+\gamma)$ at $\sqrt{s}=184,189$ and $200 \mathrm{GeV}$. The RACOON results are taken from ref. [5].

\begin{tabular}{|c|c|c|c||c|c|}
\hline Group & $\sigma_{B}^{\text {full }}$ & $\sigma_{V+S+H}$ & $\sigma_{t o t}$ & $\sigma_{V}^{\text {fact }}$ & $\sigma_{V+S+H^{-}} \sigma_{V}^{\text {fact }}$ \\
\hline KKS(zero-width) & $1980.4(4)$ & -178.9 & 1801.5 & -3441.7 & 3261.6 \\
KKS & $1980.4(4)$ & -172.8 & 1807.6 & -3323.2 & 3150.4 \\
RACOON & $1980.8(7)$ & -183.4 & 1797.4 & -3318.2 & 3134.8 \\
\hline
\end{tabular}

Table 6. The full-Born cross section, $\sigma_{B}^{\text {full }}$, as well as the radiative correction, $\sigma_{V+S+H}$, adding up to the total cross section, $\sigma_{t o t}$, for the process $e^{+} e^{-} \rightarrow W^{+} W^{-} \rightarrow(u, \bar{d})(\bar{c} s)(+\gamma)$ at $\sqrt{s}=200 \mathrm{GeV}$. The factorizable part of the virtual correction, $\sigma_{V}^{\text {fact }}$, as well as the difference, $\sigma_{V+S+H^{-}} \sigma_{V}^{\text {fact }}$, for $m_{\gamma}=10^{-6} \mathrm{GeV}$ is also shown.

\begin{tabular}{|c|c|l|l|l|l|l|l|}
\hline Group & $\sigma_{B}^{\text {DPA }}$ & \multicolumn{2}{|c|}{$\sigma_{V}$} & \multicolumn{2}{c|}{$\sigma_{S}\left(E_{\gamma}<0.1\right)$} & \multicolumn{2}{c|}{$\sigma_{H}\left(E_{\gamma}>0.1\right)$} \\
\cline { 3 - 8 } & & fact. & non-fact. & fact. & non-fact. & fact. & non-fact. \\
\hline \multirow{2}{*}{ KKS } & $1994.7(1)$ & $-3323.2(4)$ & & $1695.5(3)$ & 46.2 & 1454.4 & \\
\cline { 3 - 8 } & & \multicolumn{2}{|c|}{$1741.7(3)$} & & \\
\hline \multirow{2}{*}{$\begin{array}{c}\text { KKS } \\
\text { (lab.) }\end{array}$} & $1991.7(3)$ & $-3317.3(6)$ & & & & & \\
\cline { 3 - 8 } & & \multicolumn{2}{|c|}{-3377.4} & \multicolumn{2}{c|}{1739.2} & \multicolumn{2}{|c|}{1454.8} \\
\hline
\end{tabular}

Table 7. Comparison of $\sigma_{V}, \sigma_{S}$ and $\sigma_{H}$ for $e^{+} e^{-} \rightarrow W^{+} W^{-} \rightarrow(u \bar{d})(\bar{d} s)$ at $\sqrt{s}=200$ $\mathrm{GeV}$ for $k_{c}=0.1 \mathrm{GeV}$ and for $m_{\gamma}=10^{-6} \mathrm{GeV}$. (Note that the slight difference between the sum, $\sigma_{V+S+H}$, calculated from the results in this Table, and $\sigma_{V+S+H}$ in Table 6 , is due to the somewhat larger value of $k_{c}$ ).

In Tables 5 to 7 , our results are compared with the ones from RACOON. According to Tables 5 and 6 , the observed pattern of deviations between KKS, KKS(zero-width) and RACOON is similar to the one found in the case of the leptonic $W^{ \pm}$decays: 
i) The results for $\sigma_{\text {tot }}$ from KKS at $\sqrt{s}=200 \mathrm{GeV}$ lie about $10 \mathrm{fb}$ or $0.55 \%$ above the ones from RACOON.

ii) The results from KKS(zero-width), which simulate the zero-width treatment employed by BBC, are only $3 f b$ or $0.17 \%$ larger than the ones from RACOON. As discussed under i) and iii) for the leptonic $W^{ \pm}$ decay, the zero-width approximation decreases the total cross section, thus leading to an (accidental) coincidence of the RACOON and KKS(zerowidth) result.

Remembering Table 1, the difference between KKS and RACOON is expected to be due to the difference in the treatment of the photon radiation (DPA versus radiation from the full set of Born diagrams) and to the different choice of the fermion angles kept invariant under the on-shell continuation in $k_{ \pm}^{2}$. In addition, we have to keep in mind that RACOON, as a consequence of the complexity of their program, did not explicitly verify the cancellation of the non-factorizable corrections in their calculation of $\sigma_{V+S+H}$.

In the ensuing interpretation of the difference between RACOON and KKS, we proceed in two steps:

i) We assume that the result by RACOON, even though being obtained by including the non-factorizable corrections to $\sigma_{V}, \sigma_{S}$ and $\sigma_{H}$, implicitly contains a cancellation of these contributions in the sum, $\sigma_{V+S+H}$. The last column of Table 6 then shows that the factorizable contribution to photon radiation, $\sigma_{S+H}^{\text {fact }}$, by KKS exceeds the one by RACOON by 15 $f b$. The difference in $\sigma_{V}^{\text {fact }}$ amounts to $-5 f b$. Altogether this adds up to the mentioned difference of $10 \mathrm{fb}$ in $\sigma_{\text {tot }}$.

In order to clarify the origin of the difference of $5 f b$ in $\sigma_{V}^{\text {fact }}$, between KKS and RACOON, we look at Table 7. In Table 7, in addition to the results from KKS and RACOON, we provide results (i.e. KKS (lab.)) for $\sigma_{B}^{\mathrm{DPA}}$ and $\sigma_{V}^{\text {fact }}$ employing laboratory fermion angles, as used by RACOON in their treatment of $\sigma_{V}$. The use of the laboratoryframe fermion angles decreases the KKS results for $\sigma_{V}^{\text {fact }}$ by $0.15 \%$ to agree with the ones from RACOON. The difference of $5 \mathrm{fb}$ in $\sigma_{V}^{\text {fact }}$ is accordingly traced back to the different choice of the fermion angles kept constant under extrapolation in $k_{ \pm}^{2}$ ฯ

ii) Alternatively, let us assume that the different treatment of the photon radiation by KKS and by RACOON nevertheless leads to identical

\footnotetext{
${ }^{6}$ In [i], the results from RACOON are also compared with the results from YFSWW, by Jadach et al., whereby also the dominant higher-order radiative corrections are included. The results by YFSWW lie up to about $0.4 \%$ above the ones from RACOON.

${ }^{7}$ The absolute value of $\sigma_{V}^{\text {fact }}$ being dependent on the choice of the regularizing photon mass, the comparison of $\sigma_{V}^{\text {fact }}$ of KKS and RACOON is carried out for technical reasons and is meant to explicitly demonstrate the consistency of the results under identical boundary conditions.
} 
results for the factorizable part of the photon radiation, i.e. we assume that the factorizable part of the photon radiation, even though unknown to the RACOON treatment, can be well represented by the KKS results. For $\sigma_{S}$, such a hypothesis might be suggested by the excellent agreement of KKS and RACOON for $\sigma_{S}$ given in Table 7. Under this assumption, the non-factorizable part of the radiative corrections, implicitly contained in the RACOON result, can be calculated from the difference of the KKS and RACOON results for $\sigma_{V}, \sigma_{S}$ and $\sigma_{H}$. According to Table 7, it becomes

$$
\begin{aligned}
\sigma_{V+S+H}^{\text {non-fact }} & =\sigma_{V}^{\text {non-fact }}+\sigma_{S}^{\text {non-fact }}+\left(\sigma_{H}^{\mathrm{RACOON}}-\sigma_{H}^{\text {fact }}\right) \\
& =-12.6 \mathrm{fb} .
\end{aligned}
$$

Under the above hypothesis that the magnitude of the factorizable part of the photon radiation is unaffected by replacing the full set of diagrams by the reduced set of the DPA, we thus arrive at the conclusion that the RACOON result for $\sigma_{V+S+H}$ contains a non-factorizable contribution to the photon radiation that is not cancelling the nonfactorizable contribution to $\sigma_{V}$. This non-factorizable contribution of about $-12 f b$ would then be responsible for the difference in $\sigma_{t o t}$ of about $-10 f b$ between KKS and RACOON.

Actually, both the hypotheses discussed in i) and ii) may be considered as extreme cases. A detailed analysis of the RACOON calculation may indeed reveal that both, the different treatment of the radiation as well as some mismatch of non-factorizable corrections, are at the root of the $0.6 \%$ difference of the results of KKS and RACOON on $\sigma_{t o t}$.

\section{Conclusions}

By explicitly simulating the results of BBC and RACOON, whenever possible, we found excellent agreement with BBC and RACOON. Due to the complexity of the calculations, this in itself is a remarkable result. Differences among the final results up to about $0.6 \%$ were traced back to the different underlying implementations of the double-pole approximation. Roughly speaking, the different treatment of the photon radiation (by using the DPA) leads to an increase of our results relative to the ones of RACOON. This increase is (partially) cancelled, if the additional simplifying approximation of on-shell kinematics (the BBC zero-width approximation) is adopted. As a consequence of the known difficulties concerning the treatment of unstable particles, it remains an open question, to what extent even a technically extremely demanding full-one-loop evaluation (without DPA) could possibly reduce theoretical uncertainties. 


\section{Acknowledgement}

We would like to thank S. Dittmaier and S. Chapovsky for communicating their numerical results to us, and for providing details on the structure of their computational procedures. We would like to also thank J. Fujimoto and T.Ishikawa for valuable discussions and suggestions.

\section{References}

[1] Reports of the working groups on precision calculations for LEP2 physics, eds, S. Jadach, G. Passarino and R. Pittau, CERN 2000-009, hep-ph/0005309.

[2] Y. Kurihara, M. Kuroda and D. Schildknecht, Nucl. Phys. B565(2000) 49.

[3] W.Beenakker, F.A. Berends and A.P. Chapovsky, Nucl.Phys. B548(1999)3.

[4] A. Denner, S. Dittmaier, M. Roth and D. Wackeroth, Nucl.Phys.B560 (1999) 33.

[5] A. Denner, S. Dittmaier, M. Roth and D. Wackeroth, Phys.Lett. B475 (2000) 127, hep-ph/9912261.

[6] A. Denner, S. Dittmaier, M. Roth and D. Wackeroth, Nucl.Phys. B587 (2000) 67, hep-ph/0006307.

[7] V.S. Fadin, V.A. Khoze and A.D. Martin, Phys. Rev. D49 (1994) 2447.

[8] W. Beenakker, F.A.Berends and A.P. Chapovsky, Phys. Lett. B411 (1997) 203; Nucl. Phys. B508(1997) 17.

[9] A. Denner, S. Dittmaier, M.Roth and D. Wackeroth, Phys. Lett. B429( 1998) 145; Nucl. Phys. B519 (1998) 39.

[10] A.P. Chapovsky and V.A.Khoze, E.J. of Phys. 9(1999)499.

[11] S. Kawabata, Comp. Commun. Phys. 41(1986) 127, ibid 77 (1995) 309.

[12] T. Ishikawa et al., GRACE manual, ver.1.0, 1993.

[13] M. Kuroda, Analytic expression of the radiative corrections to the process $e^{+} e^{-} \rightarrow W^{+} W^{-}$in one-loop order, 1994, unpublished. 